\title{
Desempleo y salud: Un análisis de la repercusión de la crisis económica sobre la salud de los españoles
}

\author{
ROSA M. URBANOS-GARRIDO a ${ }^{\text {a }}$ BEATRIZ GONZÁLEZ LÓPEZ-VALCÁRCEL ${ }^{\text {b }}$ \\ a Universidad Complutense de Madrid, Facultad CC.EE., Campus de Somosaguas, s/n, 28223 \\ Pozuelo de Alarcón (Madrid), España.E-mail: urbanos@ccee.ucm.es \\ ${ }^{b}$ Universidad de Las Palmas de Gran Canarias, Facultad CC.EE., Campus de Tafira, 35017 Las \\ Palmas de Gran Canaria, España.E-mail: bvalcarcel@dmc.ulpgc.es
}

\begin{abstract}
RESUMEN
En este trabajo se exploran las consecuencias de la crisis económica sobre la salud de los adultos españoles con especial énfasis en los efectos del desempleo, a partir del modelo conceptual de Dahlgren y Whitehead. Se analizan microdatos longitudinales de la Encuesta de Condiciones de Vida (ECV) 2006 a 2011 y los microdatos transversales de la Encuesta Nacional de Salud de España (ENSE) 2011-12, empleando modelos econométricos para explicar el nivel de salud física y mental de los individuos y sus cambios cuando varían los ingresos y la situación laboral. Los análisis de la ECV concluyen que si bien las condiciones de la vivienda y las proxies de necesidades cubiertas influyen significativamente en la salud autovalorada, ni las variaciones en la renta individual ni el paso desde la situación de empleo a estar parado tienen una influencia significativa. Los modelos basados en datos de la ENSE, por el contrario, concluyen que estar parado, una vez se controla por el resto de determinantes de la salud, tiene un impacto negativo particularmente importante sobre la salud mental y más fuerte cuanto más tiempo dura el desempleo.
\end{abstract}

Palabras clave: Desempleo, salud, modelos microeconométricos, crisis económica.

\section{Unemployment and Health: An Analysis of the Impact of the Economic Crisis on the Health of the Spanish Population}

\begin{abstract}
In this paper we explore the consequences of the economic crisis on the Spanish adult health with special emphasis on the effects of unemployment. We use the conceptual model of Dahlgren and Whitehead. We analyze longitudinal microdata from the Survey of Living Conditions (ECV) 2006-2011 and cross-sectional microdata from the National Health Survey of Spain (ENSE) 2011-12, using econometric models to explain the level of physical and mental health of individuals and their changes when varying income and employment status. Our analysis of ECV concludes that while the housing conditions and needs met proxies significantly influence self-assessed health, neither variations in individual income or the shift from employment to unemployment have a significant influence. Models based on data from the ENSE, however, conclude that after controlling for other determinants of health, unemployment has a significant negative impact on physical and mental health, and that impact is stronger the longer unemployment lasts.

Keywords: Unemployment, Health, Micreoeconometric Models, Economic Crisis.
\end{abstract}

Clasificación JEL: I12, C25, C33, E24

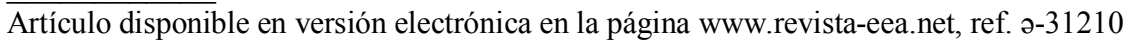




\section{INTRODUCCIÓN}

La crisis económica que España (y buena parte de los países del mundo) padece desde el año 2008, tiene su manifestación más terrible en la caída del empleo, que está conduciendo a miles de hogares a una situación próxima a la pobreza -cuando no directamente de pobreza, sin que sea posible emplear eufemismos-. En abril de 2013, la tasa de paro se situaba en el 27,16\% (INE, 2013), una cifra inédita para la economía española y que, con la excepción de Grecia, se sitúa muy lejos de la tasa correspondiente a los países de nuestro entorno geográfico y económico. Algunas previsiones auguran, incluso, un empeoramiento de esta situación, de forma que podría alcanzarse una tasa de desempleo del 27,5\% a finales de 2013, y hasta de un 28,5\% en 2014 (Diario Expansión, 2013).

Las consecuencias de un desempleo que afecta a más de la cuarta parte de la población activa tienen un reflejo evidente en la evolución económica y, desde luego, en la situación social y política del país. Pero también es de esperar que se manifiesten en el ámbito de la salud. Precisamente el objetivo de este trabajo consiste en explorar hasta qué punto el fenómeno del desempleo y, particularmente, su duración, influyen en la percepción de la salud física y mental de la población española. Para explorar la relación entre dichas variables contamos con dos tipos de bases de datos: una longitudinal, como es la Encuesta de Condiciones de Vida (ECV), que aunque diseñada para objetivos más amplios, permite relacionar desempleo y salud (general) percibida; y otra base de carácter transversal, con información más detallada sobre la salud de la población, que viene dada por la Encuesta Nacional de Salud para España en su última edición (2011-12).

El trabajo que aquí se presenta se organiza como sigue: en el apartado 2 se revisan los antecedentes más relevantes que encontramos en la literatura, y que permiten ilustrar la relación entre desempleo y salud. A continuación, en el apartado 3 se detallan los ejercicios empíricos desarrollados a partir de las dos fuentes de información citadas, y se incluyen en él tanto la descripción de la metodología como el resumen de resultados -aún provisionales, pues se derivan de una investigación en curso-. Por último, el cuarto apartado se dedica a la discusión y conclusiones.

\section{ANTECEDENTES}

A pesar de que la evidencia científica de base epidemiológica sugiere la presencia de una asociación positiva y significativa entre el desempleo y numerosos resultados de salud adversos, la relación causa efecto entre variables dista de ser evidente como consecuencia de los factores de confusión que median entre ellas, tanto de tipo clínico como social y económico (Jin et al., 1995). Además, el desempleo puede actuar positivamente sobre la salud mediante algunos me- 
canismos relacionados con el mejor empleo del tiempo, la menor exposición a riesgos de accidentes y a contaminación, entre otros. Un estudio reciente (Stuckler et al., 2009), comprueba con datos de 26 Estados de la UE que el aumento en las tasas de desempleo se asocia con incrementos significativos a corto plazo en el número de muertes violentas prematuras, pero también con una reducción de las víctimas de tráfico. Hay varias publicaciones en revistas de economía que concluyen que las crisis económicas tienen un papel contracíclico en términos de salud y que en países ricos el desempleo a corto plazo mejora la salud de las poblaciones y reduce la mortalidad general y por determinadas causas (Ruhm, 2000; Gerdtham y Ruhm, 2006). De hecho, si la pérdida de PIB durante las crisis económicas de los últimos cincuenta años se ajustara por el valor estadístico de las vidas humanas "ahorradas", en términos de reducción de mortalidad, la volatilidad de las pérdidas de PIB en EEUU se reduciría un 37\% y un $46 \%$ para otros países (Egan et al., 2013).

La relación causa-efecto entre desempleo y salud es, a priori, bidireccional: la hipótesis de la "exposición al trabajo" se centra en los efectos que el desempleo tiene sobre la salud/enfermedad de las personas, mientras que la hipótesis de "selección" señala cómo una pobre salud aumenta el riesgo de caer en desempleo. A pesar de que ambas hipótesis se han debatido largamente (Winefield, 1995; Novo et al., 2000), pocos estudios empíricos controlan por la posible endogeneidad que la inclusión de la variable desempleo puede implicar en la estimación de los modelos econométricos de salud. En todo caso, cuando se controla este efecto se comprueba que la relación causal inversa, si bien explica una parte de las diferencias observadas en las tasas de mortalidad de empleados y parados, no puede dar cuenta de todas las diferencias halladas entre ambos grupos (Stewart, 2001). En consecuencia, el efecto "exposición" parece revelarse como más importante en comparación con el debido a la "selección" (EMCONET, 2007), e incluso se ha comprobado que puede ser más fuerte una vez que se controla por el efecto "selección" (Claussen, 1999; Novo, 2000; Hammarström y Janlert, 2002). En línea con esta hipótesis se encuentran abundantes trabajos que ponen de manifiesto que el empleo protege y fomenta la salud (entre otros, Ross y Mirowsky, 1995; Dooley et al., 1996; Platt et al., 1999; Kasl y Jones, 2000).

Los mecanismos mediadores entre desempleo y salud/enfermedad son, sin duda, complejos y multidimensionales, y vienen explicados a partir de distintos modelos teóricos. Así, los modelos de privación económica suponen que el desempleo conduce a los parados a una situación económica que tiende a empeorar los pre-requisitos para la salud (Janlert, 1991). El desempleo tiene como efecto inmediato una reducción de ingresos que puede manifestarse en dificultades para mantener una dieta nutritiva y adecuada, y por tanto, impactar en la salud de los individuos. Paradójicamente las caídas de renta, derivadas del desempleo o no, pueden causar obesidad en países desarrollados, donde los 
precios relativos de los alimentos obesogénicos son mas altos y tienden a aumentar mas que los de las frutas y verduras frescas. El precio de la caloría es inversamente proporcional a la densidad calórica. La dieta mas económica es la más obesogénica, y la que siguen por necesidad los más pobres (Darmon y Drewnowski, 2008; Drewnowski y Specter, 2004). El problema ya no es sólo de salud, es de gradiente social y de equidad en la medida en que la obesidad afecta más a los más desfavorecidos. Asimismo, una reducción en el nivel de renta puede disminuir el nivel de atención médica que las personas buscan al caer enfermas, como consecuencia de las dificultades para hacer frente a los gastos asociados al tratamiento (World Bank, 2009). Este efecto es particularmente acusado en los países en los que la cobertura de los seguros de salud está vinculada al empleo o basada en financiación privada (co-pagos elevados) (Dávila y González, 2009).

Por su parte, de acuerdo con los "modelos de estrés" (Kagan y Levi, 1975), el desempleo y la incertidumbre acerca de la situación laboral futura pueden actuar como un factor estresante que conduzca a cambios psicológicos, a alteraciones de los hábitos de vida con impacto en la salud y, en definitiva, a una salud más deteriorada. Por ejemplo, el desempleo y la caída en la renta se asocian a un consumo excesivo de alcohol (Mossakowski, 2008). No obstante, la literatura describe cómo los mecanismos de estrés pueden manifestarse en efectos de signo contrario (Catalano et al., 2011). Así, existe evidencia que muestra cómo las economías en declive aumentan la incidencia de acontecimientos estresantes relacionados con el empleo y con la situación financiera de los individuos, que aumentan la probabilidad de experimentar otros factores de estrés no asociados de forma intuitiva con la evolución económica (como las dificultades familiares/maritales) (Catalano et al., 1987; Vinokur et al., 1996). En el mismo sentido, algunos trabajos sugieren que las recesiones económicas fomentan que los individuos anticipen situaciones estresantes, que incluyen la pérdida del empleo y la dificultad para hacer frente a las obligaciones financieras (Karasek y Theorell, 1990; y este mismo estudio). Sin embargo, si se parte del hecho de que una parte importante del estrés diario procede del puesto de trabajo (Karasek y Theorell, 1990), una reducción del tiempo dedicado a trabajar puede reducir la prevalencia de enfermedades inducidas por el estrés. Asimismo, es de esperar que las enfermedades asociadas a los riesgos laborales se reduzcan, bien por la pérdida del empleo, bien porque un ritmo de trabajo más lento permite prestar más atención a las cuestiones relacionadas con la seguridad (Boone y van Ours, 2009; Davies et al., 2009; entre otros).

Paul y Moser (2009) ponen de manifiesto en un metanálisis reciente la asociación entre desempleo (y su duración) y salud mental, incluyendo más de 300 estudios publicados hasta 2008. Además de certificar la mayor prevalencia de problemas como el estrés, la depresión, la ansiedad, o la falta de autoestima entre los parados, comprueban que el efecto del desempleo en la salud mental es 
mayor en países con menor desarrollo económico, mayor desigualdad de la renta y sistemas de protección social al desempleo más débiles. De su revisión de estudios de carácter longitudinal y experimentos naturales se deduce asimismo que el desempleo, no sólo está correlacionado con las situaciones de angustia, sino que también la causa.

En tercer lugar, el denominado "modelo de apoyo social" se vincula estrechamente con el modelo de estrés, e implica que el desempleo lleva a un aumento del aislamiento social que puede derivar en un impacto directo sobre la salud o en un menor efecto amortiguador del apoyo (Roberts et al., 1997). Otros modelos ponen el acento en la falta de control sobre la propia vida que se deriva de la situación de desempleo, y que también puede tener consecuencias negativas para la salud (Karasek y Theorell, 1990). Finalmente, el denominado "modelo de función latente", se basa en las necesidades (más allá de las puramente económicas) que ayuda a satisfacer un puesto de trabajo (estructura horaria del día, experiencias compartidas y contactos con otras personas, estatus, identidad, y búsqueda de objetivos colectivos) (EMCONET, 2007).

Los efectos del desempleo en la salud pueden depender del plazo considerado en el análisis. De acuerdo con los resultados obtenidos por Brenner (1979, 1983, 1987) las tasas de desempleo con frecuencia van asociadas a un descenso de la mortalidad en el corto plazo, si bien en el largo plazo tienden a asociarse a incrementos en la tasa de mortalidad, siendo el efecto neto sobre los indicadores de salud muy negativo. El efecto a largo plazo puede explicarse como consecuencia de que las tasas de desempleo de hoy se manifiestan en caídas de la renta que persisten durante más de una década. Sullivan y von Wachter (2009) estiman para USA que el aumento de la mortalidad debida al desempleo puede persistir hasta 20 años después de la pérdida del empleo, y derivar en una caída media de la esperanza de vida entre un año y un año y medio. Dao y Loungani (2010) sugieren que esta persistencia a largo plazo, cuando la pérdida de renta debida al desempleo es de gran magnitud y prolongada en el tiempo, se registra incluso en países con Estados de bienestar generosos y bajas desigualdades salariales.

El desempleo, en todo caso, no es más que una de las caras de la relación crisis económica-salud, y su efecto depende de forma crítica del entorno en que se produce. Así, está documentado que la caída del empleo y de la renta que se ha producido en Islandia en la crisis que comenzó en 2008 ha llevado a una reducción de algunos comportamientos poco saludables, como el consumo de tabaco, alcohol, bebidas azucaradas y comida rápida, o el bronceado artificial (Laufey et al., 2012). Sin duda el efecto del desempleo en la salud es función del contexto nacional, como apuntan Suhrcke y Stuckler (2012). La sensibilidad de la morbi-mortalidad a la crisis depende de las diferencias en la protección social que provee cada Estado. Pero todo apunta a que el aumento en las cifras de paro 
que se está registrando como consecuencia de la crisis muy probablemente tendrá consecuencias negativas en la salud de los europeos.

Hay una creciente evidencia de que países como Grecia, muy afectados por la crisis económica y los recortes, están registrando un aumento de los suicidios y de los brotes de enfermedades infecciosas (Karanikolos et al., 2013). Se sugiere que, aunque las recesiones suponen un riesgo para la salud per se, es la interacción entre la austeridad fiscal (en un contexto de shock económico contractivo) y la protección social débil lo que tiende a intensificar las crisis de salud en Europa. España también está adoptando medidas estrictas de austeridad fiscal que podrían poner en riesgo la salud a corto y a largo plazo. Está documentado que en las fases de recesión económica tienden a aumentar los suicidios entre los varones españoles (Tapia Granados, 2005). Y durante la actual crisis se ha registrado un aumento significativo de las consultas de atención primaria debidas a estados de ansiedad, depresión y abuso del alcohol, que en al menos un 50\% se atribuyen al efecto combinado del desempleo y de las dificultades para hacer frente a los pagos hipotecarios (Gili et al., 2013).

\section{ANÁLISIS DE LA RELACIÓN DESEMPLEO-SALUD PARA LA POBLACIÓN ESPAÑOLA}

\subsection{Análisis de la relación desempleo-salud a partir de la Encuesta de Condiciones de Vida (2007-2010)}

La Encuesta de Condiciones de Vida (ECV), elaborada anualmente por el INE $^{1}$ con metodología común con el resto de países de la UE ("European Statistics on Income and Living Conditions") proporciona datos longitudinales de una muestra representativa de hogares españoles. La salud autopercibida y la presencia de enfermedades crónicas se registran, así como un detallado conjunto de cuestiones relacionadas con la situación socioeconómica de los hogares, con énfasis en el estudio de distribución de ingresos y la exclusión social. En este trabajo utilizamos los microdatos longitudinales suministrados por la ECV durante los años 2007 a 2010. Hemos seleccionado a los individuos adultos que se encuentran activos durante al menos dos de los años de seguimiento, sea en situación de ocupados o de desempleados. El tamaño de la muestra es de 12.873 individuos, seguidos longitudinalmente dos años (5.998), tres años (4.159) o 4 años (2.716), que hacen un total de 35.337 observaciones.

Hemos estimado un modelo de panel de efectos fijos para los individuos, donde la variable dependiente viene dada por la autovaloración del estado de salud. Esta variable se codifica con valores del 1 al 5, que se aplican a la escala muy malo-muy bueno, correspondiendo el valor más alto al mejor estado de

\footnotetext{
${ }^{1}$ http://www.ine.es/daco/daco42/condivi/ecv_metodo.pdf
} 
salud posible. Hemos calculado el estimador intragrupos, ajustando las estimaciones de los errores estándar por las correlaciones intragrupo (estimadores robustos). El estimador intragrupos se basa en la información sobre los cambios en las $\mathrm{X}$ de un año a otro para el mismo individuo. Las características individuales y familiares que no cambian (o apenas cambian) en el tiempo (sexo, nivel de estudios), o que lo hacen linealmente de forma determinista (la edad), determinan conjuntamente el nivel basal de salud (la constante individual de cada persona).

La variable explicativa de mayor interés es la dicotómica "parado", que vale 1 si en la semana anterior a la encuesta la persona declaraba estar en paro, y 0 si estaba ocupado, trabajando o de baja laboral.

Las otras variables explicativas son la renta individual, monetaria o cuasimonetaria del asalariado el año anterior a la entrevista, en euros anuales (renta_individual), y se incluyen también en el modelo varias proxies sobre la situación económica familiar y la capacidad de los sujetos para cubrir sus necesidades, así como sobre las condiciones de habitabilidad de la vivienda. Así, la dummy "imprevistos" toma valor 1 cuando la familia puede hacer frente a gastos imprevistos, y la variable "fin_mes", que indica si tiene capacidad para llegar a fin de mes, se define mediante una escala ordinal con las categorías "Con mucha dificultad" (1), "Con dificultad" (2), "Con cierta dificultad" (3), "Con cierta facilidad" (4), "Con facilidad" (5) y "Con mucha facilidad" (6). Finalmente, la dummy "goteras" toma valor 1 cuando la vivienda registra algún problema de goteras, mientras la dummy "tempera_hogar" se iguala a 1 cuando el hogar puede mantener la vivienda a la temperatura adecuada durante el año.

La Tabla 1 presenta una descripción univariante de la muestra, diferenciando por años.

Tabla 1

Descripción univariante de la muestra de la ECV, por años

\begin{tabular}{|c|c|c|c|c|c|}
\hline & & 2007 & 2008 & 2009 & 2010 \\
\hline \multirow{5}{*}{ Salud } & Muy Mala & 0.3 & 0.3 & 0,3 & 0,3 \\
\hline & Mala & 3,0 & 2,0 & 2,2 & 2,1 \\
\hline & Regular & 15,2 & 13,1 & 14,7 & 14,2 \\
\hline & Buena & 63,9 & 67,5 & 65,3 & 65,0 \\
\hline & Muy buena & 17,5 & 17,1 & 17,5 & 18,4 \\
\hline Paro & $\%$ parados & 9,1 & 10,0 & 17,2 & 18,3 \\
\hline Renta individual & Renta anual del asalariado & $11808 €(9829)$ & $12500 €(10712)$ & $12664 €(11051)$ & $12577 €(11582)$ \\
\hline \multirow{3}{*}{$\begin{array}{l}\text { Cobertura de } \\
\text { necesidades }\end{array}$} & $\begin{array}{l}\text { Capacidad para afrontar gastos } \\
\text { imprevistos (\%) }\end{array}$ & 74,2 & 75,2 & 69,7 & 67,7 \\
\hline & $\begin{array}{l}\text { Capacidad para llegar a fin de } \\
\text { mes } 1(\%)\end{array}$ & 8,9 & 11,4 & 13,8 & 13,7 \\
\hline & $\begin{array}{l}\text { Capacidad para llegar a fin de } \\
\text { mes } 2(\%)\end{array}$ & 15,4 & 16,3 & 16,2 & 16,4 \\
\hline
\end{tabular}


Tabla 1 (continuación)

Descripción univariante de la muestra de la ECV, por años

\begin{tabular}{|c|c|c|c|c|c|}
\hline & & 2007 & 2008 & 2009 & 2010 \\
\hline \multirow{6}{*}{$\begin{array}{c}\text { Cobertura de necesidades } \\
\text { (continuación) }\end{array}$} & $\begin{array}{l}\text { Capacidad para llegar a fin de } \\
\text { mes } 3(\%)\end{array}$ & 29,6 & 29,7 & 27,4 & 26,3 \\
\hline & $\begin{array}{l}\text { Capacidad para llegar a fin de } \\
\text { mes } 4(\%)\end{array}$ & 28,0 & 27,9 & 26,6 & 26,0 \\
\hline & $\begin{array}{l}\text { Capacidad para llegar a fin de } \\
\text { mes } 5(\%)\end{array}$ & 16,5 & 13,5 & 14,6 & 16,2 \\
\hline & $\begin{array}{l}\text { Capacidad para llegar a fin de } \\
\text { mes } 6(\%)\end{array}$ & 1,6 & 1,1 & 1,3 & 1,5 \\
\hline & Goteras (\%) & 18,3 & 15,8 & 17,5 & 20,9 \\
\hline & Temperatura_hogar (\%) & 93,8 & 95,6 & 94,0 & 93,0 \\
\hline \multirow{6}{*}{$\begin{array}{c}\text { Variables } \\
\text { sociodemográficas }\end{array}$} & $\begin{array}{l}\operatorname{EDAD}\left({ }^{*}\right) \text { (el año de } \\
\text { incorporación a la muestra) }\end{array}$ & \multicolumn{4}{|c|}{$40,8(11,4)$} \\
\hline & $\%$ MUJERES & \multicolumn{4}{|c|}{44,9} \\
\hline & \% Estudios primarios o menos & \multicolumn{4}{|c|}{16,1} \\
\hline & $\begin{array}{l}\% \text { Estudios secundarios primera } \\
\text { etapa }\end{array}$ & \multicolumn{4}{|c|}{27,4} \\
\hline & $\begin{array}{l}\text { \% Estudios secundarios } \\
\text { segunda etapa }\end{array}$ & \multicolumn{4}{|c|}{23,6} \\
\hline & $\%$ Estudios superiores & \multicolumn{4}{|c|}{32,9} \\
\hline
\end{tabular}

(*) Entre paréntesis, error estándar.

Fuente: Elaboración propia a partir de los microdatos de la Encuesta de Condiciones de Vida 20072010 (INE).

Los resultados del modelo (que se muestran en la Tabla 2) indican que, si bien las condiciones de la vivienda y las proxies de necesidades cubiertas influyen significativamente en la salud autovalorada, ni las variaciones en la renta individual ni el paso desde la situación de empleo a estar parado tienen una influencia significativa en la variable dependiente. El efecto individual es muy relevante (correlación intragrupo 0,54), lo que indica que una gran parte de la salud de las personas se relaciona con sus características sociodemográficas básicas (edad, sexo, nivel de estudios).

\section{Tabla 2}

Estimaciones del modelo de efectos fijos con datos longitudinales ECV 2007-2010. Variable dependiente: nivel de salud autopercibido

(escala de 1 a 5 )

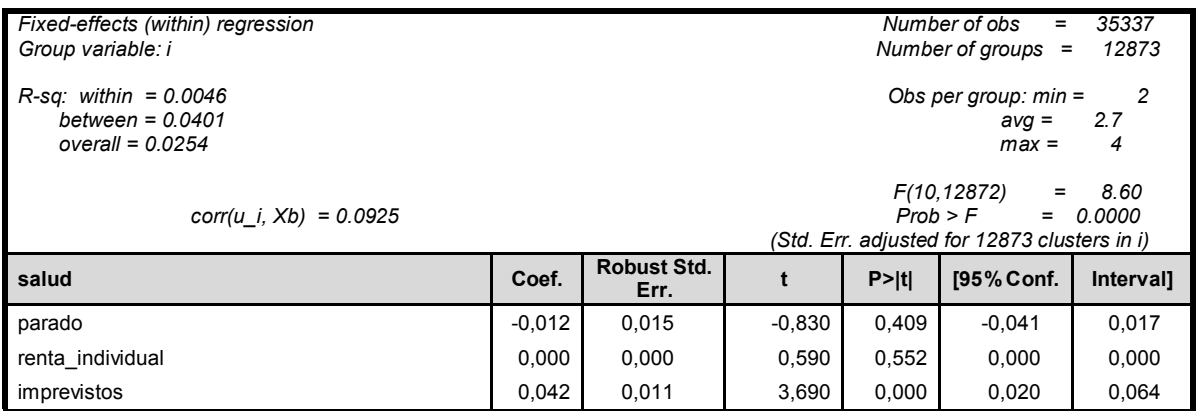




\section{Tabla 2 (continuación)}

Estimaciones del modelo de efectos fijos con datos longitudinales ECV 2007-2010.

Variable dependiente: nivel de salud autopercibido

(escala de 1 a 5 )

\begin{tabular}{|lr|r|r|r|r|r|r|}
\hline salud & & Coef. & $\begin{array}{c}\text { Robust Std. } \\
\text { Err. }\end{array}$ & $\mathbf{t}$ & $\mathbf{P}>|\mathbf{t}|$ & [95\% Conf. & Interval] \\
& 2 & 0,044 & 0,015 & 2,840 & 0,005 & 0,013 & 0,074 \\
& 3 & 0,062 & 0,016 & 3,830 & 0,000 & 0,030 & 0,094 \\
& 4 & 0,080 & 0,018 & 4,430 & 0,000 & 0,045 & 0,116 \\
& 5 & 0,085 & 0,020 & 4,180 & 0,000 & 0,045 & 0,125 \\
goteras & 6 & 0,180 & 0,037 & 4,870 & 0,000 & 0,107 & 0,252 \\
tempera_hogar & & $-0,036$ & 0,011 & $-3,180$ & 0,001 & $-0,058$ & $-0,014$ \\
const & & 0,058 & 0,017 & 3,360 & 0,001 & 0,024 & 0,093 \\
sigma_u & & 3,839 & 0,022 & 172,810 & 0,000 & 3,795 & 3,882 \\
sigma_e & & & & & & & \\
rho & & & & & & & \\
\hline
\end{tabular}

Fuente: Elaboración propia a partir de los microdatos de la Encuesta de Condiciones de Vida 2007-2010 (INE).

El modelo concluye que el desempleo no afecta a la salud autopercibida. Este resultado se aprecia ya en una simple descriptiva que cruza los cambios en el status laboral (caer o no en el desempleo de un año al siguiente) con los cambios en la salud autopercibida (Figura 1).

Figura 1

Relación entre situación laboral y cambios en el nivel de salud autopercibida

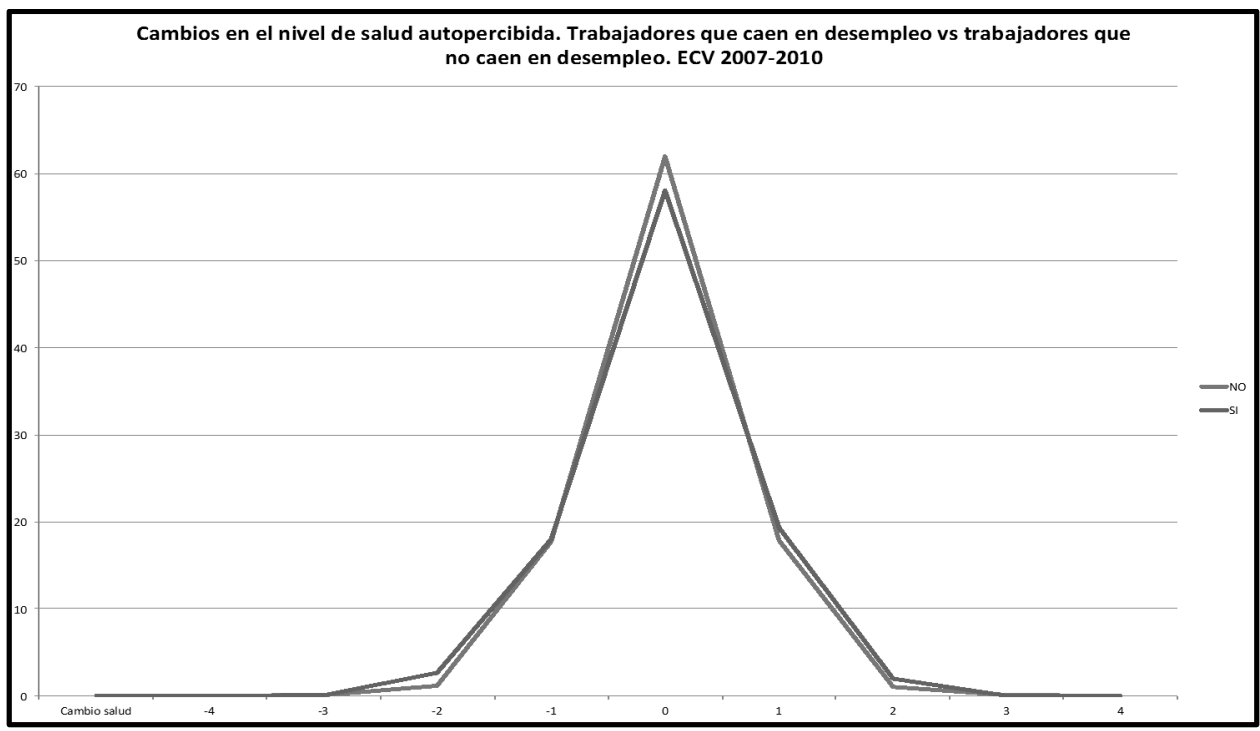

Fuente: Elaboración propia a partir de los microdatos de la ECV 2007-2010 (INE). 
Sin embargo, los cambios en las condiciones económicas del hogar sí que afectan a la salud de las personas, y a lo largo de los años del período de observación se aprecia un deterioro en dichas condiciones (Tabla 1).

\subsection{Análisis de la relación desempleo-salud a partir de la Encuesta Nacional de Salud 2011-2012}

Con datos de la ENSE 2011-2012 hemos estimado modelos para la salud, física y mental, de toda la muestra de adultos, sea cual sea su estatus laboral. La ENSE se realiza por el INE, en colaboración con el Ministerio de Sanidad, Servicios Sociales e Igualdad. Su propósito es obtener datos comparables internacionalmente sobre el estado de salud y sus factores determinantes, así como sobre la utilización de los servicios sanitarios. La muestra de la encuesta 20112012 es de aproximadamente 24.000 viviendas distribuidas en 2.000 secciones censales. La encuesta proporciona resultados nacionales y por comunidades autónomas.

Para abordar el análisis de la relación entre el desempleo y la salud seguimos el modelo conceptual de Dahlgren y Whitehead (1991), que describe los estratos de influencia en la salud a partir de un modelo social y ecológico como el que se muestra en la Figura 2. Los individuos se encuentran en el centro, con unas características no modificables (edad, sexo y genes). Rodeando al núcleo se encuentran los factores con influencia en la salud que son potencialmente modificables. El primer estrato representa los estilos de vida que pueden favorecer o dañar la salud. En segundo lugar se hallan las redes sociales y comunitarias, que pueden ofrecer apoyo a los miembros de la comunidad cuando éstos tienen problemas. A continuación se incluyen factores de tipo estructural como la vivienda, las condiciones de trabajo o el acceso a los servicios esenciales (vivienda, educación, sanidad, etc.). Por último, aparece un grupo de características más amplias relacionado con las condiciones culturales, socioeconómicas y medioambientales del entorno en el que los individuos viven.

Como indicadores de salud se consideran dos tipos de variables dependientes: por un lado, la salud general y, por otro, la salud mental. A pesar de que esta última no es sino una dimensión de la salud general, resulta particularmente susceptible de verse afectada por la situación de desempleo, tal y como se puso de manifiesto en el epígrafe 2. Por otra parte, cuando se analiza el impacto del paro sobre la salud general pueden esperarse dos efectos contrapuestos: primero, que quede difuminado el impacto habido en la salud mental, al integrarse ésta en un conjunto más amplio; segundo, que afloren problemas físicos $-\mathrm{y}$ no psíquicos o psicológicos- asociados al desempleo, que será difícil detectar cuando el análisis se circunscribe exclusivamente al ámbito de la salud mental.

En ambos casos (salud general y salud mental) se emplean dos variables aproximativas, una de carácter discreto y otra de carácter continuo. De este 
modo observaremos hasta qué punto los resultados difieren cuando se altera la forma de operativizar la salud.

Figura 2

Modelo de determinantes de la salud de Dahlgren y Whitehead

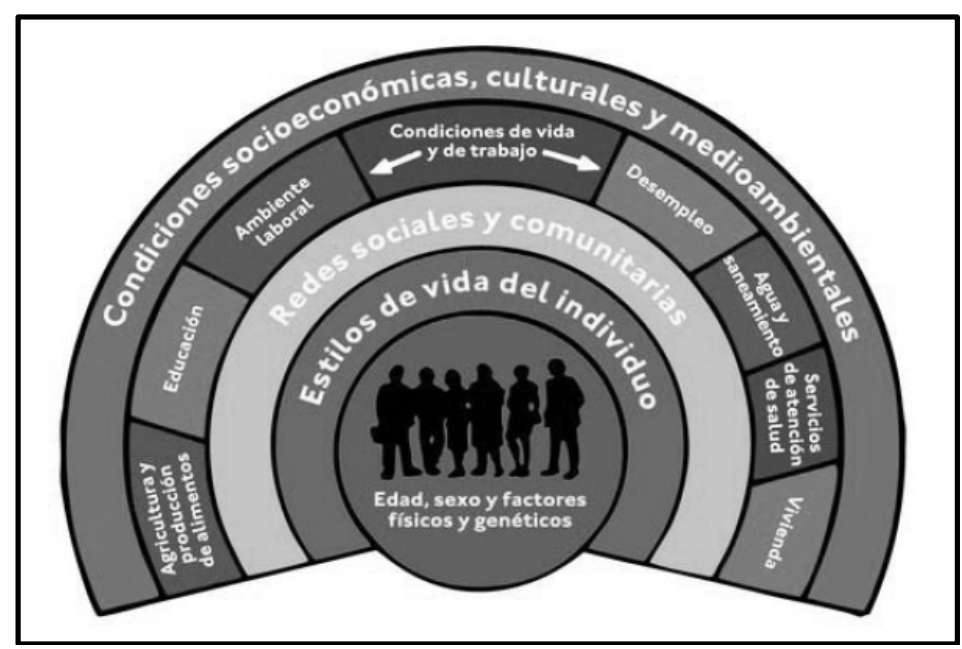

Fuente: Dahlgren y Whitehead (1991).

En lo que se refiere a la salud general, la variable discreta ( $(\mathrm{sah})$ se construye a partir de la información que suministra la pregunta clásica sobre autovaloración del estado de salud en los últimos doce meses, que se incluye en todas las ENSE. Las cinco categorías iniciales se agrupan en dos: estado de salud bueno o muy bueno vs. estado de salud regular, malo o muy malo ${ }^{2}$. Por otra parte, la variable continua (salud) se obtiene de una pregunta incluida por vez primera en la ENSE, en la que se pide al sujeto que puntúe su salud en el día de la entrevista a partir de una escala de 0 a 100 , donde el 0 denota la peor salud imaginable, y el 100 la mejor. Es la escala Visual Analógica (EVA) que forma parte del instrumento EQ5D de medida de salud ${ }^{3}$.

En lo que respecta a la salud mental, la variable discreta (mentalhealth) se obtiene de categorizar una de las dimensiones de la calidad de vida relacionada con la salud que se incluyen en el EuroQol (EQ-5D-5L), referida a la presencia

\footnotetext{
${ }^{2}$ En la literatura pueden encontrarse estudios que agrupan las categorías de otro modo, clasificando el estado de salud "regular" conjuntamente con la buena o muy buena salud. No obstante, dado que no hay consenso sobre esta cuestión y entendemos que la categoría "regular" sugiere la presencia de algún problema de salud, hemos optado por agruparla junto con las categorías de salud mala o muy mala.

${ }^{3} \mathrm{http}: / /$ www.euroqol.org
} 
de ansiedad/depresión en el último año. Finalmente, la variable continua ( $s a-$ ludmental) se define a partir de una variable derivada contenida en la propia ENSE, que refleja el índice de salud mental de Goldberg a corto plazo (Goldberg, 1978; Goldberg et al., 1997). Este índice se ha validado para cribado de riesgo de salud mental en consultas de medicina general y se emplea con frecuencia en el ámbito de la medicina clínica. Se basa en las respuestas a 12 preguntas referidas al presente ${ }^{4}$. Cada una de ellas tiene cuatro niveles, que se codifican como $0=$ "sin problema" (niveles 0 y 1 ) o $1=$ "con problema" (niveles 2 y 3 ). La variable resultante, entre 0 y 12 , se reescala invirtiéndola, de forma que sus valores oscilan entre 0 (peor salud mental, o mayor riesgo para la salud mental) y 12 (mejor salud mental, o menor riesgo).

Por su parte, el vector de variables independientes agrupa, siguiendo el modelo definido por Dahlgren y Whitehead, un conjunto de características variadas con influencia demostrada en la salud. Entre las no modificables se incluyen el sexo y la edad. Como determinantes de la salud potencialmente modificables se consideran, en primer lugar, la situación familiar de los individuos y el apoyo social que perciben. Los estilos de vida están representados por el consumo de tabaco y alcohol, la práctica de ejercicio físico y el ratio peso/talla, o Índice de Masa Corporal (como proxy de la dieta). La presencia de enfermedades crónicas se incluye como indicador de la salud preexistente de los individuos. Los determinantes socioeconómicos se representan a través del nivel de estudios y la clase social ${ }^{5}$. Las variables geográficas tratan de aproximar el efecto de los factores de tipo medioambiental, así como de las diferencias en el acceso de los sujetos a otros servicios con impacto en la salud (servicios sanitarios, sociales, infraestructuras de transporte, etc.). Por último, se incluyen las variables representativas de la relación con el mercado laboral, la situación de desempleo y su duración.

\footnotetext{
${ }^{4}$ Las preguntas son las siguientes: 1) ¿Ha podido concentrarse bien en lo que hacía? 2) ¿Sus preocupaciones le han hecho perder mucho sueño? 3) ¿Ha sentido que está desempeñando un papel útil en la vida? 4) ¿Se ha sentido capaz de tomar decisiones? 5) ¿Se ha notado constantemente agobiado y en tensión? 6) ¿Ha tenido la sensación de que no puede superar sus dificultades? 7) ¿Ha sido capaz de disfrutar de sus actividades normales de cada día? 8) ¿Ha sido capaz de hacer frente adecuadamente a sus problemas? 9) ¿Se ha sentido poco feliz o deprimido? 10) ¿Ha perdido confianza en sí mismo? 11) ¿Ha pensado que usted es una persona que no vale para nada? 12) ¿Se siente razonablemente feliz considerando todas las circunstancias? Cada una de estas preguntas tiene cuatro respuestas posibles: a) Mejor que lo habitual; b) Igual que lo habitual; c) Menos que lo habitual; y d) Mucho menos que lo habitual. A las dos primeras respuestas se les asigna, para cada pregunta, un total de 0 puntos, mientras que a las dos últimas se les asigna 1 punto.

${ }^{5}$ Desgraciadamente, la información sobre renta familiar que incluye la ENSE 2011-12 se ha eliminado de la base de datos que el Ministerio de Sanidad pone a disposición del público, por cuyo motivo no podemos explorar esta fuente de desigualdades de salud que la literatura considera tan importante.
} 
La Tabla 3 recoge con más detalle la definición de las variables empleadas en el análisis empírico, así como las características de la muestra final empleada, compuesta por un total de 17.271 individuos mayores de 16 años.

Tabla 3

Definición de variables y estadística descriptiva de la muestra de la ENSE 2011-12 $(n=17.271)$

\begin{tabular}{|c|c|c|c|}
\hline Variable & Definición & Media & $\begin{array}{l}\text { Desviación } \\
\text { estándar }\end{array}$ \\
\hline sah & $\begin{array}{l}\text { Binomial=1 si el estado de salud en los últimos } 12 \text { meses es bueno o muy } \\
\text { bueno, } 0 \text { si regular, malo o muy malo }\end{array}$ & 0,744 & 0,436 \\
\hline salud & puntuación del estado de salud en el día de la entrevista (de 0 a 100) & 78,938 & 17,768 \\
\hline mentalhealth & $\begin{array}{l}\text { Binomial =1 si el sujeto declara no estar ansioso ni deprimido, } 0 \text { en caso } \\
\text { contrario }\end{array}$ & 0,866 & 0,340 \\
\hline salud mental & $\begin{array}{l}\text { Indicador combinado de salud mental que oscila entre } 0 \text { (peor salud mental) y } \\
12 \text { (mejor salud mental). Véase nota } 2 \text {. }\end{array}$ & 10,569 & 2,577 \\
\hline mujer & 1 si mujer, 0 si hombre & 0,496 & 0,500 \\
\hline edad & Edad en años & 45,779 & 17,672 \\
\hline casado & $\begin{array}{l}1 \text { si el individuo está casado, } 0 \text { en el resto de casos (soltero, viudo, separado } \\
\text { legalmente o divorciado) }\end{array}$ & 0,576 & 0,494 \\
\hline apoyo social & $\begin{array}{l}\text { apoyo social funcional percibido: oscila entre } 11 \text { y } 55 \text {, de menor a mayor } \\
\text { apoyo }^{6}\end{array}$ & 48,576 & 7,051 \\
\hline fuma & 1 si fuma actualmente, 0 en caso contrario & 0,280 & 0,449 \\
\hline alcohol & $\begin{array}{l}\text { Consumo medio diario de alcohol semanal (lunes a domingo, en gramos de } \\
\text { alcohol puro) }\end{array}$ & 27,257 & 71,784 \\
\hline ejercicio0 & $\begin{array}{l}1 \text { si el individuo no realiza ninguna actividad física en su tiempo libre, } 0 \text { en } \\
\text { caso contrario (categoría omitida) }\end{array}$ & 0,430 & 0,495 \\
\hline ejercicio1 & $\begin{array}{l}1 \text { si realiza alguna actividad física o deportiva ocasional en su tiempo libre, } 0 \\
\text { en caso contrario }\end{array}$ & 0,334 & 0,472 \\
\hline ejercicio2 & 1 si realiza actividad física varias veces al mes, 0 en caso contrario & 0,139 & 0,346 \\
\hline ejercicio3 & 1 si hace entrenamiento deportivo o físico varias veces a la semana & 0,097 & 0,296 \\
\hline normopeso & 1 si el Índice de Masa Corporal indica normopeso, 0 en caso contrario & 0,451 & 0,498 \\
\hline crónica & $\begin{array}{l}1 \text { si el individuo padece alguna enfermedad o problema de salud crónicos o de } \\
\text { larga duración, } 0 \text { en caso contrario }\end{array}$ & 0,407 & 0,491 \\
\hline nest1 & $\begin{array}{l}1 \text { si el individuo no ha completado la educación primaria, } 0 \text { en caso contrario } \\
\text { (categoría omitida) }\end{array}$ & 0,010 & 0,101 \\
\hline nest2 & $\begin{array}{l}1 \text { si ha completado la educación primaria o la enseñanza secundaria de } \\
\text { primera etapa, } 0 \text { en caso contrario }\end{array}$ & 0,442 & 0,497 \\
\hline nest3 & $\begin{array}{l}1 \text { si ha completado estudios de bachillerato o enseñanzas profesionales de } \\
\text { grado medio o equivalentes, } 0 \text { en caso contrario }\end{array}$ & 0,232 & 0,422 \\
\hline nest4 & $\begin{array}{l}1 \text { si enseñanzas profesionales de grado superior o equivalentes, } 0 \text { en caso } \\
\text { contrario }\end{array}$ & 0,068 & 0,251 \\
\hline nest5 & 1 si estudios universitarios o equivalentes, 0 en caso contrario & 0,169 & 0,375 \\
\hline
\end{tabular}

\footnotetext{
${ }^{6}$ La variable apoyo social percibido se obtiene sumando las respuestas a cada uno de los siguientes 11 items: recibo visitas de mis amigos y familiares; recibo ayuda en asuntos relacionados con mi casa; recibo elogios y reconocimientos cuando hago bien mi trabajo; cuento con personas que se preocupan de lo que me sucede; recibo amor y afecto; tengo la posibilidad de hablar con alguien de mis problemas en el trabajo o en la casa; tengo la posibilidad de hablar con alguien de mis problemas económicos; recibo invitaciones para distraerme y salir con otras personas; recibo consejos útiles cuando me ocurre algún acontecimiento importante en mi vida; recibo ayuda cuando estoy enfermo en la cama. Las respuestas se puntúan de 1 a 5 como sigue: 1="mucho menos de lo que deseo", 2= "menos de lo que deseo"; 3 = "ni mucho ni poco"; 4 = "casi como deseo"; y 5 = "tanto como deseo".
} 
Tabla 3 (continuación)

Definición de variables y estadística descriptiva de la muestra de la ENSE 2011-12 $(n=17.271)$

\begin{tabular}{|c|c|c|c|}
\hline Variable & Definición & Media & $\begin{array}{l}\text { Desviación } \\
\text { estándar }\end{array}$ \\
\hline clase I & $\begin{array}{l}1 \text { si perteneciente a la clase social más alta (directores/as y gerentes de } \\
\text { establecimientos de } 10 \text { o más asalariados, profesionales asociados a } \\
\text { licenciaturas universitarias), } 0 \text { en caso contrario (categoría omitida) }\end{array}$ & 0,112 & 0,316 \\
\hline clase II & $\begin{array}{l}1 \text { para directores/as y gerentes de establecimientos de menos de } 10 \\
\text { asalariados, profesionales asociados a diplomaturas y otros de apoyo } \\
\text { técnico, deportistas y artistas; } 0 \text { en caso contrario }\end{array}$ & 0,076 & 0,266 \\
\hline clase III & $\begin{array}{l}1 \text { para ocupaciones intermedias y trabajadores por cuenta propia, } 0 \text { en caso } \\
\text { contrario }\end{array}$ & 0,185 & 0,388 \\
\hline clase IV & $\begin{array}{l}1 \text { para supervisores y trabajadores en ocupaciones técnicas cualificadas, } 0 \\
\text { en caso contrario }\end{array}$ & 0,154 & 0,361 \\
\hline clase V & $\begin{array}{l}1 \text { si trabajadores cualificados del sector primario y otros trabajadores } \\
\text { semicualificados, } 0 \text { en caso contrario }\end{array}$ & 0,326 & 0,469 \\
\hline clase VI & 1 si trabajadores no cualificados, 0 en caso contrario & 0,146 & 0,353 \\
\hline Andalucía & 1 si residente en Andalucía, 0 en caso contrario (categoría omitida) & 0,183 & 0,386 \\
\hline Aragón & 1 si residente en Aragón & 0,025 & 0,157 \\
\hline Asturias & 1 si residente en Asturias & 0,024 & 0,154 \\
\hline Baleares & 1 si residente en Baleares & 0,022 & 0,148 \\
\hline Canarias & 1 si residente en Canarias & 0,045 & 0,207 \\
\hline Cantabria & 1 si residente en Cantabria & 0,012 & 0,107 \\
\hline C. y León & 1 si residente en Castilla y León & 0,060 & 0,238 \\
\hline C. La Mancha & 1 si residente en Castilla-La Mancha & 0,039 & 0,194 \\
\hline Catalunya & 1 si residente en Cataluña & 0,157 & 0,364 \\
\hline C. Valenciana & 1 si residente en Comunidad Valenciana & 0,111 & 0,314 \\
\hline Extremadura & 1 si residente en Extremadura & 0,022 & 0,145 \\
\hline Galicia & 1 si residente en Galicia & 0,059 & 0,235 \\
\hline Madrid & 1 si residente en Madrid & 0,137 & 0,344 \\
\hline Murcia & 1 si residente en Murcia & 0,034 & 0,182 \\
\hline Navarra & 1 si residente en Navarra & 0,015 & 0,123 \\
\hline País Vasco & 1 si residente en País Vasco & 0,045 & 0,206 \\
\hline Rioja & 1 si residente en La Rioja & 0,007 & 0,083 \\
\hline Ceuta & 1 si residente en Ceuta & 0,002 & 0,039 \\
\hline Melilla & 1 si residente en Melilla & 0,001 & 0,038 \\
\hline trabaja & 1 si el individuo trabaja, 0 en caso contrario (categoría omitida) & 0,464 & 0,499 \\
\hline inactivo & $\begin{array}{l}1 \text { si el individuo está inactivo (estudiantes, jubilados, discapacitados, } \\
\text { dedicados a las labores del hogar y otros), } 0 \text { en caso contrario }\end{array}$ & 0,388 & 0,487 \\
\hline parado0 & $\begin{array}{l}1 \text { si parado y no ha trabajado nunca } \\
0 \text { en caso contrario }\end{array}$ & 0,007 & 0,081 \\
\hline parado1 & $\begin{array}{l}1 \text { si en paro menos de } 6 \text { meses } \\
0 \text { en caso contrario }\end{array}$ & 0,047 & 0,213 \\
\hline parado2 & $\begin{array}{l}1 \text { si en paro de } 6 \text { meses a } 1 \text { año } \\
0 \text { en caso contrario }\end{array}$ & 0,023 & 0,149 \\
\hline parado3 & $\begin{array}{l}1 \text { si en paro de } 1 \text { año a menos de } 2 \\
0 \text { en caso contrario }\end{array}$ & 0,028 & 0,164 \\
\hline parado4 & $\begin{array}{l}1 \text { si en paro durante más de } 2 \text { años } \\
0 \text { en caso contrario }\end{array}$ & 0,045 & 0,207 \\
\hline
\end{tabular}

Fuente: Elaboración propia a partir de los microdatos de la Encuesta Nacional de Salud DE 20112012 (INE).

Los modelos con variables dependientes dicotómicas se estiman mediante sendos probit, mientras que cuando las variables representativas de la salud son de tipo continuo los modelos se estiman mediante mínimos cuadrados ordina- 
rios ajustando por heterocedasticidad los estimadores de los errores estándar. Las Tablas 4-7 muestran los resultados de las estimaciones para las cuatro variables dependientes consideradas.

Tabla 4

Estimación de la autovaloración del estado de salud

\begin{tabular}{|c|c|c|c|c|c|c|}
\hline $\begin{array}{l}\text { Number of obs } \\
\text { Wald chi2(44) } \\
\text { Prob }>\text { chi2 }= \\
\text { Log pseudolike }\end{array}$ & $-7447,643$ & & Pseudo R2 & $=0,2419$ & & \\
\hline sah & $\mathrm{dF} / \mathrm{dx}$ & $\begin{array}{l}\text { Robust } \\
\text { Std. Err }\end{array}$ & $\mathbf{z}$ & $P>|z|$ & {$[90 \%$} & C.I.] \\
\hline mujer & $-0,0403$ & 0,0086 & $-4,660$ & 0,000 & $-0,0545$ & $-0,0261$ \\
\hline edad & $-0,0042$ & 0,0003 & $-13,600$ & 0,000 & $-0,0047$ & $-0,0037$ \\
\hline casado & 0,0105 & 0,0086 & 1,230 & 0,220 & $-0,0036$ & 0,0247 \\
\hline apoyo social & 0,0047 & 0,0006 & 8,420 & 0,000 & 0,0038 & 0,0056 \\
\hline fuma & $-0,0326$ & 0,0099 & $-3,360$ & 0,001 & $-0,0489$ & $-0,0164$ \\
\hline alcohol & 0,0000 & 0,0001 & 0,330 & 0,752 & $-0,0001$ & 0,0001 \\
\hline ejercicio1 & 0,0640 & 0,0083 & 7,440 & 0,000 & 0,0504 & 0,0776 \\
\hline ejercicio2 & 0,0922 & 0,0115 & 6,930 & 0,000 & 0,0733 & 0,1111 \\
\hline ejercicio3 & 0,0906 & 0,0125 & 6,190 & 0,000 & 0,0701 & 0,1111 \\
\hline normopeso & 0,0312 & 0,0086 & 3,600 & 0,000 & 0,0170 & 0,0453 \\
\hline crónica & $-0,2867$ & 0,0089 & $-32,200$ & 0,000 & $-0,3013$ & $-0,2722$ \\
\hline nest2 & 0,0429 & 0,0130 & 3,280 & 0,001 & 0,0215 & 0,0644 \\
\hline nest3 & 0,0755 & 0,0140 & 5,030 & 0,000 & 0,0525 & 0,0985 \\
\hline nest4 & 0,1116 & 0,0141 & 6,260 & 0,000 & 0,0884 & 0,1349 \\
\hline nest5 & 0,1051 & 0,0145 & 6,300 & 0,000 & 0,0813 & 0,1289 \\
\hline clase II & $-0,0448$ & 0,0221 & $-2,130$ & 0,033 & $-0,0812$ & $-0,0085$ \\
\hline clase III & $-0,0381$ & 0,0177 & $-2,220$ & 0,026 & $-0,0672$ & $-0,0090$ \\
\hline clase IV & $-0,0414$ & 0,0194 & $-2,220$ & 0,027 & $-0,0732$ & $-0,0095$ \\
\hline clase V & $-0,0740$ & 0,0176 & $-4,340$ & 0,000 & $-0,1029$ & $-0,0450$ \\
\hline clase VI & $-0,0892$ & 0,0216 & $-4,430$ & 0,000 & $-0,1247$ & $-0,0537$ \\
\hline Aragón & 0,0250 & 0,0214 & 1,130 & 0,260 & $-0,0101$ & 0,0601 \\
\hline Asturias & $-0,0158$ & 0,0203 & $-0,790$ & 0,429 & $-0,0492$ & 0,0177 \\
\hline Baleares & 0,0626 & 0,0190 & 2,930 & 0,003 & 0,0313 & 0,0939 \\
\hline Canarias & $-0,0692$ & 0,0226 & $-3,270$ & 0,001 & $-0,1064$ & $-0,0320$ \\
\hline Cantabria & 0,1064 & 0,0155 & 5,310 & 0,000 & 0,0810 & 0,1318 \\
\hline C. y León & 0,0186 & 0,0162 & 1,120 & 0,262 & $-0,0080$ & 0,0453 \\
\hline C. La Mancha & 0,0585 & 0,0163 & 3,260 & 0,001 & 0,0317 & 0,0852 \\
\hline Catalunya & 0,0362 & 0,0142 & 2,450 & 0,014 & 0,0129 & 0,0595 \\
\hline C. Valenciana & $-0,0050$ & 0,0167 & $-0,300$ & 0,762 & $-0,0325$ & 0,0224 \\
\hline Extremadura & 0,0297 & 0,0190 & 1,490 & 0,136 & $-0,0016$ & 0,0610 \\
\hline Galicia & $-0,0329$ & 0,0197 & $-1,730$ & 0,083 & $-0,0653$ & $-0,0005$ \\
\hline Madrid & 0,0349 & 0,0150 & 2,230 & 0,026 & 0,0102 & 0,0597 \\
\hline Murcia & 0,0162 & 0,0198 & 0,800 & 0,424 & $-0,0164$ & 0,0487 \\
\hline Navarra & 0,0487 & 0,0174 & 2,580 & 0,010 & 0,0201 & 0,0773 \\
\hline País Vasco & 0,0238 & 0,0170 & 1,350 & 0,176 & $-0,0042$ & 0,0518 \\
\hline Rioja & 0,0243 & 0,0218 & 1,080 & 0,281 & $-0,0114$ & 0,0601 \\
\hline Ceuta & 0,0176 & 0,0384 & 0,450 & 0,656 & $-0,0455$ & 0,0807 \\
\hline Melilla & 0,0014 & 0,0364 & 0,040 & 0,969 & $-0,0584$ & 0,0612 \\
\hline inactivo & $-0,0228$ & 0,0103 & $-2,230$ & 0,025 & $-0,0397$ & $-0,0059$ \\
\hline parado0 & 0,0541 & 0,0529 & 0,920 & 0,357 & $-0,0329$ & 0,1411 \\
\hline parado1 & 0,0018 & 0,0226 & 0,080 & 0,936 & $-0,0353$ & 0,0390 \\
\hline parado2 & $-0,0423$ & 0,0325 & $-1,370$ & 0,172 & $-0,0958$ & 0,0112 \\
\hline parado3 & $-0,0448$ & 0,0264 & $-1,790$ & 0,074 & $-0,0882$ & $-0,0013$ \\
\hline parado4 & $-0,0696$ & 0,0228 & $-3,280$ & 0,001 & $-0,1071$ & $-0,0321$ \\
\hline
\end{tabular}

Fuente: Elaboración propia. 
Tabla 5

Estimación del estado de salud general (variable continua)

\begin{tabular}{|c|c|c|c|c|c|c|}
\hline $\begin{array}{l}\text { Number of obs } \\
F(44,17226)= \\
\text { Prob }>F= \\
\text { R-squared = }\end{array}$ & & Root MSE & $=14,88$ & & & \\
\hline Salud & Coef. & $\begin{array}{l}\text { Robust } \\
\text { Std. Err }\end{array}$ & $\mathbf{t}$ & $P>|t|$ & {$[90 \%$} & C.I.] \\
\hline mujer & $-1,0894$ & 0,3245 & $-3,360$ & 0,001 & $-1,6232$ & $-0,5556$ \\
\hline edad & $-0,2342$ & 0,0110 & $-21,250$ & 0,000 & $-0,2523$ & $-0,2161$ \\
\hline casado & 0,6806 & 0,3146 & 2,160 & 0,031 & 0,1631 & 1,1980 \\
\hline apoyo social & 0,1779 & 0,0230 & 7,740 & 0,000 & 0,1401 & 0,2157 \\
\hline fuma & $-1,2354$ & 0,3270 & $-3,780$ & 0,000 & $-1,7733$ & $-0,6976$ \\
\hline alcohol & 0,0006 & 0,0002 & 3,040 & 0,002 & 0,0003 & 0,0009 \\
\hline ejercicio1 & 3,4548 & 0,3369 & 10,250 & 0,000 & 2,9006 & 4,0090 \\
\hline ejercicio2 & 3,8219 & 0,3967 & 9,640 & 0,000 & 3,1694 & 4,4743 \\
\hline ejercicio3 & 4,0007 & 0,4673 & 8,560 & 0,000 & 3,2321 & 4,7694 \\
\hline normopeso & 1,5747 & 0,3083 & 5,110 & 0,000 & 1,0675 & 2,0819 \\
\hline crónica & $-10,6380$ & 0,3286 & $-32,380$ & 0,000 & $-11,1785$ & $-1,0097$ \\
\hline nest2 & 2,8544 & 0,6016 & 4,740 & 0,000 & 1,8647 & 3,8440 \\
\hline nest3 & 3,6061 & 0,6699 & 5,380 & 0,000 & 2,5041 & 4,7081 \\
\hline nest4 & 4,3234 & 0,7821 & 5,530 & 0,000 & 3,0368 & 5,6099 \\
\hline nest5 & 3,5313 & 0,7191 & 4,910 & 0,000 & 2,3484 & 4,7142 \\
\hline clase II & $-0,5155$ & 0,5945 & $-0,870$ & 0,386 & $-1,4934$ & 0,4624 \\
\hline clase III & $-0,6992$ & 0,4984 & $-1,400$ & 0,161 & $-1,5191$ & 0,1207 \\
\hline clase IV & $-0,3071$ & 0,5626 & $-0,550$ & 0,585 & $-1,2325$ & 0,6183 \\
\hline clase V & $-0,8716$ & 0,5069 & $-1,720$ & 0,086 & $-1,7055$ & $-0,0377$ \\
\hline clase VI & $-1,3201$ & 0,6076 & $-2,170$ & 0,030 & $-2,3196$ & $-0,3206$ \\
\hline Aragón & $-0,1340$ & 0,7030 & $-0,190$ & 0,849 & $-1,2904$ & 1,0224 \\
\hline Asturias & $-0,7845$ & 0,6885 & $-1,140$ & 0,255 & $-1,9170$ & 0,3480 \\
\hline Baleares & 1,5398 & 0,9266 & 1,660 & 0,097 & 0,0156 & 3,0640 \\
\hline Canarias & $-1,1437$ & 0,8060 & $-1,420$ & 0,156 & $-2,4695$ & 0,1821 \\
\hline Cantabria & $-7,1260$ & 0,8123 & $-8,770$ & 0,000 & $-8,4621$ & $-5,7899$ \\
\hline C. y León & 0,1912 & 0,6193 & 0,310 & 0,758 & $-0,8275$ & 1,2098 \\
\hline C. La Mancha & 1,1982 & 0,6821 & 1,760 & 0,079 & 0,0762 & 2,3202 \\
\hline Catalunya & $-0,1084$ & 0,5529 & $-0,200$ & 0,845 & $-1,0178$ & 0,8011 \\
\hline C. Valenciana & 2,8956 & 0,6100 & 4,750 & 0,000 & 1,8922 & 3,8989 \\
\hline Extremadura & 4,5675 & 0,7322 & 6,240 & 0,000 & 3,3632 & 5,7719 \\
\hline Galicia & $-0,0376$ & 0,5727 & $-0,070$ & 0,948 & $-0,9797$ & 0,9045 \\
\hline Madrid & 4,2595 & 0,5357 & 7,950 & 0,000 & 3,3782 & 5,1407 \\
\hline Murcia & 4,6506 & 0,7262 & 6,400 & 0,000 & 3,4561 & 5,8451 \\
\hline Navarra & $-0,0141$ & 0,6429 & $-0,020$ & 0,982 & $-1,0716$ & 1,0433 \\
\hline País Vasco & 0,8675 & 0,6155 & 1,410 & 0,159 & $-0,1450$ & 1,8799 \\
\hline Rioja & 2,6834 & 0,6871 & 3,910 & 0,000 & 1,5532 & 3,8135 \\
\hline Ceuta & 0,6449 & 1,5836 & 0,410 & 0,684 & $-1,9600$ & 3,2498 \\
\hline Melilla & $-0,7466$ & 1,3994 & $-0,530$ & 0,594 & $-3,0485$ & 1,5554 \\
\hline inactivo & $-2,1884$ & 0,3496 & $-6,260$ & 0,000 & $-2,7635$ & $-1,6133$ \\
\hline parado0 & 2,6478 & 1,1336 & 2,340 & 0,020 & 0,7830 & 4,5125 \\
\hline parado1 & 0,9455 & 0,6565 & 1,440 & 0,150 & $-0,1345$ & 2,0254 \\
\hline parado2 & $-0,6393$ & 0,9229 & $-0,690$ & 0,488 & $-2,1575$ & 0,8788 \\
\hline parado3 & $-1,6435$ & 0,8409 & $-1,950$ & 0,051 & $-3,0268$ & $-0,2602$ \\
\hline parado4 & $-1,6533$ & 0,7876 & $-2,100$ & 0,036 & $-2,9488$ & $-0,3579$ \\
\hline _cons & 80,2198 & 1,5705 & 51,080 & 0,000 & 77,6363 & 82,8032 \\
\hline
\end{tabular}

Fuente: Elaboración propia. 
Tabla 6

Estimación del estado de salud mental (variable dicotómica)

\begin{tabular}{|c|c|c|c|c|c|c|}
\hline $\begin{array}{l}\text { Number of obs }= \\
\text { Wald chi2 }(44)=1 \\
\text { Prob }>\text { chi2 }=0, \\
\text { Log pseudolikelih }\end{array}$ & $\begin{array}{l}1 \\
17 \\
-5765,725\end{array}$ & & & $=0,1510$ & & \\
\hline Mentalhealth & $d F / d x$ & $\begin{array}{l}\text { Robust } \\
\text { Std. Err }\end{array}$ & $\frac{\text { Pseudo R2 }}{\mathbf{z}}$ & $P>|z|$ & {$[90 \%$} & C.I.] \\
\hline mujer & $-0,0541$ & 0,0060 & $-8,880$ & 0,000 & $-0,0640$ & $-0,0442$ \\
\hline edad & $-0,0014$ & 0,0002 & $-6,750$ & 0,000 & $-0,0017$ & $-0,0010$ \\
\hline casado & 0,0114 & 0,0060 & 1,920 & 0,055 & 0,0016 & 0,0212 \\
\hline apoyo social & 0,0047 & 0,0004 & 12,700 & 0,000 & 0,0041 & 0,0053 \\
\hline fuma & $-0,0354$ & 0,0073 & $-5,050$ & 0,000 & $-0,0474$ & $-0,0233$ \\
\hline alcohol & 0,0000 & 0,0000 & 0,980 & 0,326 & $-0,0000$ & 0,0001 \\
\hline ejercicio1 & 0,0377 & 0,0056 & 6,420 & 0,000 & 0,0285 & 0,0469 \\
\hline ejercicio2 & 0,0260 & 0,0094 & 2,540 & 0,011 & 0,0106 & 0,0414 \\
\hline ejercicio3 & 0,0492 & 0,0081 & 4,930 & 0,000 & 0,0358 & 0,0625 \\
\hline normopeso & 0,0117 & 0,0061 & 1,920 & 0,055 & 0,0017 & 0,0217 \\
\hline crónica & $-0,1218$ & 0,0068 & $-18,690$ & 0,000 & $-0,1330$ & $-0,1107$ \\
\hline nest2 & 0,0053 & 0,0089 & 0,590 & 0,552 & $-0,0093$ & 0,0199 \\
\hline nest3 & 0,0142 & 0,0104 & 1,330 & 0,183 & $-0,0028$ & 0,0313 \\
\hline nest4 & 0,0272 & 0,0124 & 1,980 & 0,048 & 0,0068 & 0,0476 \\
\hline nest5 & 0,0257 & 0,0114 & 2,110 & 0,035 & 0,0070 & 0,0444 \\
\hline clase II & $-0,0259$ & 0,0155 & $-1,790$ & 0,073 & $-0,0513$ & $-0,0005$ \\
\hline clase III & $-0,0001$ & 0,0119 & $-0,010$ & 0,993 & $-0,0196$ & 0,0194 \\
\hline clase IV & 0,0103 & 0,0119 & 0,840 & 0,399 & $-0,0092$ & 0,0298 \\
\hline clase V & $-0,0044$ & 0,0118 & $-0,370$ & 0,711 & $-0,0238$ & 0,0151 \\
\hline clase VI & $-0,0317$ & 0,0152 & $-2,240$ & 0,025 & $-0,0566$ & $-0,0067$ \\
\hline Aragón & 0,0212 & 0,0138 & 1,410 & 0,158 & $-0,0016$ & 0,0440 \\
\hline Asturias & $-0,0435$ & 0,0172 & $-2,810$ & 0,005 & $-0,0718$ & $-0,0151$ \\
\hline Baleares & 0,0005 & 0,0164 & 0,030 & 0,975 & $-0,0265$ & 0,0275 \\
\hline Canarias & $-0,0383$ & 0,0162 & $-2,600$ & 0,009 & $-0,0650$ & $-0,0116$ \\
\hline Cantabria & 0,0692 & 0,0089 & 4,830 & 0,000 & 0,0546 & 0,0839 \\
\hline C. y León & 0,0299 & 0,0102 & 2,630 & 0,008 & 0,0132 & 0,0466 \\
\hline C. La Mancha & 0,0250 & 0,0123 & 1,850 & 0,065 & 0,0048 & 0,0452 \\
\hline Catalunya & $-0,0339$ & 0,0122 & $-2,970$ & 0,003 & $-0,0540$ & $-0,0138$ \\
\hline C. Valenciana & $-0,0086$ & 0,0119 & $-0,740$ & 0,462 & $-0,0282$ & 0,0110 \\
\hline Extremadura & 0,0338 & 0,0111 & 2,640 & 0,008 & 0,0155 & 0,0521 \\
\hline Galicia & $-0,0336$ & 0,0144 & $-2,540$ & 0,011 & $-0,0573$ & $-0,0099$ \\
\hline Madrid & $-0,0166$ & 0,0123 & $-1,410$ & 0,159 & $-0,0369$ & 0,0036 \\
\hline Murcia & 0,0351 & 0,0115 & 2,620 & 0,009 & 0,0161 & 0,0540 \\
\hline Navarra & $-0,0222$ & 0,0168 & $-1,410$ & 0,159 & $-0,0499$ & 0,0054 \\
\hline País Vasco & 0,0125 & 0,0123 & 0,970 & 0,331 & $-0,0078$ & 0,0327 \\
\hline Rioja & 0,0179 & 0,0157 & 1,060 & 0,287 & $-0,0079$ & 0,0436 \\
\hline Ceuta & 0,0308 & 0,0231 & 1,160 & 0,247 & $-0,0071$ & 0,0688 \\
\hline Melilla & $-0,0379$ & 0,0446 & $-0,950$ & 0,344 & $-0,1112$ & 0,0355 \\
\hline inactivo & $-0,0245$ & 0,0072 & $-3,480$ & 0,001 & $-0,0364$ & $-0,0127$ \\
\hline parado0 & 0,0224 & 0,0349 & 0,580 & 0,561 & $-0,0350$ & 0,0798 \\
\hline parado1 & $-0,0391$ & 0,0187 & $-2,310$ & 0,021 & $-0,0699$ & $-0,0083$ \\
\hline parado2 & $-0,1182$ & 0,0290 & $-5,070$ & 0,000 & $-0,1660$ & $-0,0704$ \\
\hline parado3 & $-0,1027$ & 0,0261 & $-4,830$ & 0,000 & $-0,1457$ & $-0,0598$ \\
\hline parado4 & $-0,1238$ & 0,0245 & $-6,340$ & 0,000 & $-0,1641$ & $-0,0834$ \\
\hline
\end{tabular}

Fuente: Elaboración propia. 
Tabla 7

Estimación del estado de salud mental (variable continua)

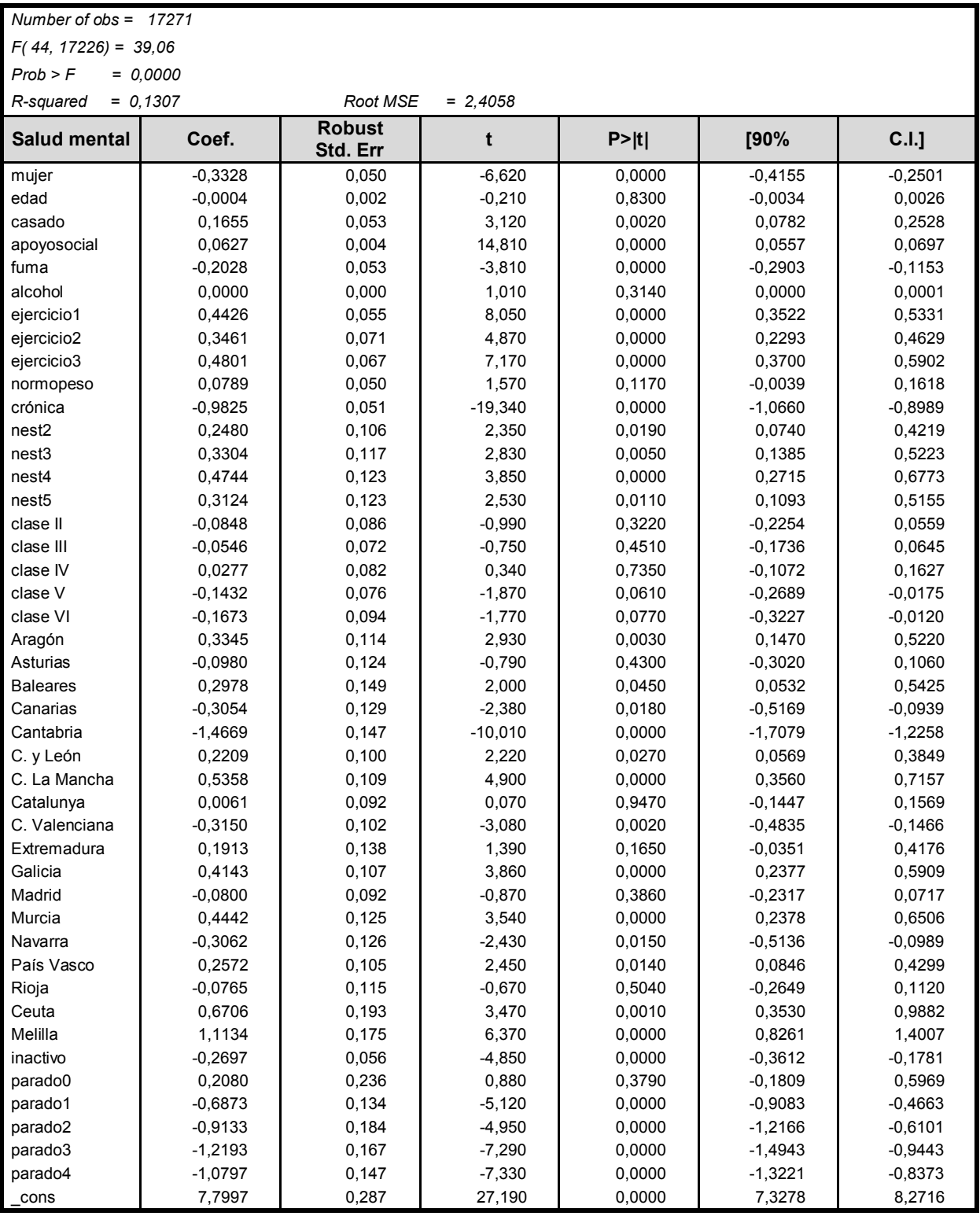

Fuente: Elaboración propia.

Los resultados son muy concordantes entre modelos, salvo para los coeficientes de las CCAA, comparadas con Andalucía. Los signos son los esperados 
y la mayor parte de los determinantes de salud resultan significativos. La situación familiar afecta de modo apreciable sólo a la salud mental, pero el apoyo social percibido se muestra como un componente influyente tanto en la salud mental como en la general. En lo que respecta a los estilos de vida, la práctica de ejercicio físico muestra indudables efectos positivos en todos los modelos estimados. Por el contrario, el consumo de tabaco redunda sistemáticamente en una peor salud, con independencia de cómo se aproxime ésta. La educación se confirma una vez más como un factor estrechamente vinculado al estado de salud (física y mental), pero la clase social (basada en la ocupación) sólo indica un gradiente social significativo cuando se estima la variable binaria de salud general. La Tabla 8 resume los signos de las variables significativas al 5\% en los cuatro modelos.

Centrándonos ya en el efecto de las variables relacionadas con el empleo, parece claro que estar parado, una vez se controla por el resto de determinantes de la salud, tiene un impacto negativo particularmente importante sobre la salud mental. Por otra parte, los resultados mostrados en las Tablas 4-7 sugieren que la duración del desempleo también constituye un factor significativo en la explicación de las diferencias en el estado de salud (física y mental) de los adultos españoles. En general, cuanto más tiempo permanece en paro un individuo, más intensamente se manifiestan los efectos negativos sobre el estado de salud. Estar parado durante más de 2 años está asociado de forma significativa a una peor salud (en relación con estar ocupado) en los cuatro modelos. Y en el caso de la salud general, donde el impacto del desempleo es menos intenso, el efecto empieza a ser significativo (al 10\%) cuando las personas llevan para-das más de 1 año.

Tabla 8

Resumen de los modelos. Signo de las variables significativas al $5 \%$

\begin{tabular}{|c|c|c|c|c|}
\hline \multirow[t]{2}{*}{ Variable } & \multicolumn{2}{|c|}{ Salud física (general) } & \multicolumn{2}{|c|}{ Salud mental } \\
\hline & $\begin{array}{l}\text { Salud física } \\
\text { binaria }\end{array}$ & Salud física continua & $\begin{array}{l}\text { Salud mental binaria } \\
\text { (largo plazo) }\end{array}$ & $\begin{array}{c}\text { Salud mental } \\
\text { continua (riesgo a } \\
\text { corto plazo) }\end{array}$ \\
\hline mujer & - & - & - & - \\
\hline edad & - & - & - & \\
\hline casado & & + & & + \\
\hline apoyo social & + & + & + & + \\
\hline fuma & - & - & - & - \\
\hline alcohol & & + & & \\
\hline ejercicio 0 & Ref. & Ref. & Ref. & Ref. \\
\hline ejercicio1 & + & + & + & + \\
\hline ejercicio2 & + & + & + & + \\
\hline ejercicio3 & + & + & + & + \\
\hline normopeso & + & + & & \\
\hline crónica & - & - & - & - \\
\hline nest1 & Ref. & Ref. & Ref. & Ref. \\
\hline nest2 & + & + & & + \\
\hline nest3 & + & + & & + \\
\hline nest4 & + & + & + & + \\
\hline nest5 & + & + & + & + \\
\hline
\end{tabular}


Tabla 8 (continuación)

Resumen de los modelos. Signo de las variables significativas al 5\%

\begin{tabular}{|c|c|c|c|c|}
\hline \multirow[t]{2}{*}{ Variable } & \multicolumn{2}{|c|}{ Salud física (general) } & \multicolumn{2}{|c|}{ Salud mental } \\
\hline & $\begin{array}{c}\text { Salud física } \\
\text { binaria }\end{array}$ & Salud física continua & $\begin{array}{l}\text { Salud mental binaria } \\
\text { (largo plazo) }\end{array}$ & $\begin{array}{c}\text { Salud mental } \\
\text { continua (riesgo a } \\
\text { corto plazo) }\end{array}$ \\
\hline clase I & Ref. & Ref. & Ref. & Ref. \\
\hline clase II & - & & & \\
\hline clase III & - & & & \\
\hline clase IV & - & & & \\
\hline clase V & - & & & \\
\hline clase $\mathrm{VI}$ & - & - & - & \\
\hline Andalucía & Ref. & Ref. & Ref. & Ref. \\
\hline Aragón & & & & + \\
\hline Asturias & & & - & \\
\hline Baleares & + & & & + \\
\hline Canarias & - & & - & - \\
\hline Cantabria & + & - & - & - \\
\hline C. y León & & & + & + \\
\hline C. La Mancha & + & & & + \\
\hline Catalunya & + & & - & \\
\hline C. Valenciana & & + & & - \\
\hline Extremadura & & + & + & \\
\hline Galicia & & & - & + \\
\hline Madrid & + & + & & \\
\hline Murcia & & + & + & + \\
\hline Navarra & + & & & - \\
\hline País Vasco & & & & + \\
\hline Rioja & & + & & \\
\hline Ceuta & & & & + \\
\hline Melilla & & & & + \\
\hline trabaja & Ref. & Ref. & Ref. & Ref. \\
\hline inactivo & - & - & - & - \\
\hline parado0 & & + & & \\
\hline parado1 & & & - & - \\
\hline parado2 & & & - & - \\
\hline parado3 & & & - & - \\
\hline parado4 & - & - & - & - \\
\hline
\end{tabular}

Fuente: Elaboración propia.

\section{DISCUSIÓN Y CONCLUSIONES}

El análisis aquí presentado trata de aportar alguna evidencia de cómo el fenómeno del desempleo puede afectar a la salud de los españoles, en un contexto en el que la tasa de paro alcanza cifras insospechadamente elevadas. Para ello se han utilizado datos procedentes de dos fuentes de información que hemos considerado complementarias.

El empleo de la ECV para un trabajo de estas características tiene ventajas e inconvenientes. Del lado de las ventajas no puede obviarse el carácter longitudinal de los datos, y el amplio detalle sobre las condiciones laborales de los individuos. Del lado de los inconvenientes, en cambio, es preciso mencionar la escasa información que esta encuesta suministra sobre el estado de salud de los entrevistados (que se limita a la salud autovalorada y a la presencia de enfermedades crónicas y limitantes), así como sobre otro tipo de variables con influencia demostrada en la salud (por ejemplo, los estilos de vida). Por ese motivo la 
relación desempleo-salud se ha explorado también a partir de los datos que suministra la Encuesta Nacional de Salud.

El análisis de los datos de la ECV, para la muestra de individuos activos, concluye que caer en desempleo no empeora la salud autopercibida. Del análisis de la ENSE, por el contrario, se desprende que el desempleo se asocia con una peor salud general y, sobre todo, mental. Además, la duración del desempleo resulta relevante para explicar las diferencias en salud de la población, particularmente en aquellos casos en los que el paro sobrepasa el año. Estos resultados son consistentes con alguna evidencia previa que, si bien confirma la existencia de una relación negativa entre desempleo y salud autovalorada con datos de sección cruzada, la desmiente cuando se emplean datos longitudinales (Böckerman et al., 2009).

En todo caso, tanto la ECV como la ENSE nos ofrecen una visión limitada de cómo la crisis (y particularmente la relacionada con el mercado de trabajo) repercutirá en la salud de los españoles. La relación desempleo-salud debería enfocarse desde una perspectiva de ciclo vital, si bien las bases de datos disponibles no suelen facilitar las investigaciones con ese enfoque. Por ejemplo, existe evidencia acerca de cómo el desempleo acumulado entre los 16 y los 30 años subyace al gradiente social en salud observado tanto entre hombres como entre mujeres (EMCONET, 2007). Pero por el momento no podemos explorar este tipo de relaciones para España.

La lectura de los resultados obtenidos permite confirmar algunos temores, y también apuntar algunas recomendaciones. En una situación como la actual, en la que ni siquiera se avista el final de la crisis económica, las políticas públicas deberían concentrarse en prevenir que la salud se deteriore en los grupos vulnerables, como los desempleados y otros grupos de bajo nivel socioeconómico (Suhrcke y Stuckler, 2012), y también en prevenir riesgos de salud específicos que afloran en las recesiones, como los problemas psicológicos.

Hay que tener en cuenta, además, que es precisamente la población más vulnerable la que se ve más afectada tanto por el aumento del desempleo como por los recortes en el sistema sanitario público que van asociados a las políticas de consolidación fiscal actuales. En ausencia de acciones que traten de controlar de algún modo los efectos de la crisis sobre estas personas, es de esperar que las desigualdades sociales (también en salud) sigan aumentando como ya lo vienen haciendo en los últimos años. 


\section{REFERENCIAS BIBLIOGRÁFICAS}

BÖCKERMAN P., ILMAKUNNAS P. (2009). Unemployment and self-assessed health: evidence from panel data. Health Economics, 18: 161-179.

BOONE J, VAN OURS JC. (2006). Are recessions good for workplace safety? J. Health Econ. 25:1069-93

BRENNER, M.H. (1979). "Mortality and the national economy. A review, and the experience of England and Wales, 1936-76". Lancet, 2(8142): 568-573.

BRENNER, M.H. (1983). "Mortality and economic instability: detailed analyses for Britain and comparative analyses for selected industrialized countries". International Journal of Health Services, 13(4): 563-620.

BRENNER, M.H. (1987). "Economic instability, unemployment rates, behavioral risks, and mortality rates in Scotland, 1952-1983". International Journal of Health Services, 17(3): 475-487.

CATALANO, R., DOOLEY, D., ROOK K. (1987). "A test of reciprocal risk between undesirable economic and noneconomic life events". Am. J. Community Psychol., 15:633-51.

CATALANO, R., GOLDMAN-MELLOR, S., SAXTON, K., et al. (2011). "The Health Effects of Economic Decline". Annual Review of Public Health, 32:43150.

CLAUSSEN, B. (1999). "Alcohol disorders and re-employment in a 5-year followup of long-term unemployed". Addiction, 94(1):133-8.

DAHLGREN, G., WHITEHEAD, M. (1991). Policies and strategies to promote social equity in health. Institute for Future Studies, Stockholm (Mimeo).

DAO, M., LOUNGANI, P. (2010). The Human Cost of Recessions: Assessing It. IMF Staff Position Note, November 11, SPN/10/17.

DARMON, N., DREWNOWSKI, A. (2008). "Does social class predict diet quality?". Am J Clin Nutr.; 87(5):1107-17. Epub 2008/05/13.

DAVIES, R., JONES, P., NÚÑEZ, I. (2009). "The impact of the business cycle on occupational injuries in the UK". Soc. Sci. Med., 69: 178-82.

DÁVILA, C.D., GONZÁLEZ, B. (2009). "Crisis económica y salud”. Gaceta Sanitaria, 23, 261-265.

DIARIO EXPANSIÓN (2013). Fitch prevé una tasa de paro en España del 28,5\% en 2014. Publicado el 3 de mayo de 2013. Disponible en URL: http://www.expansion.com/2013/05/03/economia/1367586803.html

DREWNOWSKI, A., SPECTER, S.E. (2004). "Poverty and obesity: the role of energy density and energy costs". Am J Clin Nutr.;79(1):6-16. Epub 2003/12/20.

DOOLEY, D., FIELDING, J., LEVI, L. (1996). "Health and unemployment”. Annual Review of Public Health, 17: 949-965. 
EGAN MARK, L., MULLIGAN, C.B., PHILIPSON, T.J. (2013). "Adjusting Measures of Economic Output for Health: Is the Business Cycle Countercyclical?". NBER Working Paper No. 19058, May 2013.

EMPLOYMENT CONDITIONS KNOWLEDGE NETWORK (EMCONET) (2007). Employment conditions and health inequalities. Final Report to the WHO Commission on Social Determinants of Health.

GERDTHAM, U.G., RUHM, C.J. (2006). "Deaths rise in good economic times: evidence from the OECD". Economics \& Human Biology, 4(3): 298-316.

GILI, M., ROCA, M., BASU, S., MCKEE, M., et al., (2013). "The mental health risks of economic crisis in Spain: evidence from primary care centres, 2006 and 2010". European Journal of Public Health, 23: 103-08.

GOLDBERG, D. (1978). "Mental health priorities in a primary care setting". Ann N Y Acad Sci; 310: 65-68.

GOLDBERG, D.P., GATER, R., et al., (1997). "The validity of two versions of the GHQ in the WHO study of mental illness in general health care". Psychol Med; 27(1): 191-197.

HAMMARSTRÖM, A., JANLERT, U. (2002). "Early unemployment can contribute to adult health problems - results from a longitudinal study of school-leavers". Int J Epidemiology and Community Health, 56 (8): 624-630.

INE (varios años). Encuesta de Condiciones de Vida.

INE (2013). Nota de prensa. Encuesta de Población Activa (EPA). Primer trimestre de 2013. Publicada el 25 de abril de 2013. Disponible en URL: http://www.ine.es/daco/daco42/daco4211/epa0113.pdf

INE y Ministerio de Sanidad, Servicios Sociales e Igualdad (2013). Encuesta Nacional de Salud de España 2011-12.

JANLERT, U. (1991). Work deprivation and health. Consequences of job loss and unemployment. Stockholm:Karolinska Institute.

JIN, R.L., SHAH, C.P., et al., (1995). "The impact of unemployment on health: a review of the evidence". CMAJ;153(5): 529-540.

KAGAN, A., LEVI, L. (1975). "Health and environment-psychosocial stimuli. A review". En: Levi L (Ed). Society stress and disease. Vol II. London: Oxford University Press 241-260.

KARANIKOLOS, M., MLADOVSKY, P., CYLUS, J., et al., (2013). "Financial crisis, austerity, and health in Europe". Lancet, Published online March 27, 2013 http://dx.doi.org/10.1016/S0140-6736(13)60102-6 1.

KARASEK, R., THEORELL, T. (1990). Healthy Work: Stress, Productivity, and the Reconstruction of Working Life. New York: Basic Books.

KASL, S., JONES, B. (2000). "The impact of job loss and retirement on health". En: L. Berkman, \& I. Kawachi (Eds.), Social epidemiology (pp. 118-136). Oxford: Oxford University Press. 
LAUFEY, T., CORMAN, H., NOONAN, K., et al., (2012). "Are Recessions Good For Your Health Behaviors? Impacts Of The Economic Crisis In Iceland". NBER Working Paper 18233.

MOSSAKOWSKI, K.N. (2008). "Is the duration of poverty and unemployment a risk factor for heavy drinking?". Social Science \& Medicine, 67: 947-955.

NOVO, M. (2000). Young and unemployed - does the trade cycle matter for the health. A study of young men and women during times of prosperity and times of recession. Umeå: Umeå Universitet.

PAUL, K.I., MOSER, K. (2009). "Unemployment impairs mental health: Metaanalyses". Journal of Vocational Behavior, 74: 264-282.

PLATT, S., PAVIS, S., AKRAM, G. (1999). Changing labour market conditions and health: A systematic literature review (1993-98). Research Unit in Health and Behavioural Change, University of Edinburgh Medical School, Edinburgh.

ROBERTS, H., PEARSON, J.C., MADELEY, R.J., HANFORD, S., MAGOWAN, R. (1997). "Unemployment and health: the quality of social support among residents in the Trent region of England". J Epidemiol Community Health; 51(1):41-5.

ROSS, C., MIROWSKY, J. (1995). "Does employment affect health?". Journal of Health and Social Behavior, 36: 230-243.

RUHM, C.J. (2000). "Are recessions good for your health?". The Quarterly Journal of Economics, 115(2): 617-650.

STEWART, J.M. (2001). "The impact of health status on the duration of unemployment spells and the implications for studies of the impact of unemployment on health status". Journal of Health Economics, 20: 781-796.

STUCKLER, D., BASU, S., SUHRCKE, M., COUTTS, A., MCKEE, M. (2009). "The public health effect of economic crises and alternative policy responses in Europe: an empirical analysis". Lancet 374: 315-23

SUHRCKE, M., STUCKLER, D. (2012). "Will the recession be bad for our health? It depends". Social Science \& Medicine, 74: 647-653.

SULLIVAN, D., VON WACHTER, T. (2009). "Job Displacement and mortality: An Analysis using Administrative Data”. Quarterly Journal of Economics, August 2009.

TAPIA GRANADOS, J.A. (2005). "Recessions and Mortality in Spain, 19801997". European Journal of Population, 21: 393-422.

VINOKUR, A.D., PRICE, R.H., CAPLAN, R.D. (1996). "Hard times and hurtful partners: how financial strain affects depression and relationship satisfaction of unemployed persons and their spouses". J. Pers. Soc. Psychol. 71:166-79.

WINEFIELD, A.H. (1995). "Unemployment: Its psychological costs". International Review of Industrial and Organizational Psychology, 10: 169-212.

WORLD BANK (2009). Averting A Human Crisis During the Global Downturn. Washington. 